\title{
Metodologias Ativas de Aprendizagem: construção de diagnósticos de enfermagem sobre um estudo de caso
}

\author{
Active Methods of learning: construction of diagnostics of nursing in a case study \\ Metodologías de aprendizaje activo: construcción de diagnósticos de enfermería en un \\ estudio de caso
}

\begin{abstract}
Glenda Keyla China Quemel ${ }^{1 *}$, Dayane Azevedo Maia1, Caroline Martins da Silva Moia1, Claudiane Santana Silveira Amorim ${ }^{1}$, Aloma Sena Soares ${ }^{1}$, Cássio Freitas dos Santos ${ }^{1}$, Tatyellen Natasha da Costa Oliveira¹, Márcia Geovanna Araújo Paz¹.
\end{abstract}

\section{RESUMO}

Objetivo: Relatar a experiência de graduandos em Enfermagem na construção de Diagnósticos de Enfermagem para um estudo de caso de paciente com Lepstospirose. Métodos: Trata-se de um estudo descritivo, qualitativo, tipo relato de experiência, realizado durante a disciplina "Doenças Infecciosas e Parasitárias" do curso de Graduação em Enfermagem de uma Universidade Pública, em Belém-PA. Os discentes selecionaram um tema sobre as patologias prevalentes na Amazônia, para realizarem os Diagnósticos de Enfermagem. As autoras ficaram incumbidas de elencar os diagnósticos para a patologia Leptospirose mediante exame clínico do paciente. Resultados: A partir do estudo de caso proposto em sala de aula, foram selecionados os seguintes "Diagnósticos de Enfermagem" com base na NANDA-I: Risco para infecção; Risco de termoregulação ineficaz; Deambulação prejudicada; Nutrição desequilibrada: menor do que as necessidades corporais; Dor aguda. Considerações finais: A utilização de metodologias ativas nas instituições de ensino contribui para formação crítica-reflexiva de futuros Enfermeiros, os quais estarão mais aptos a desenvolveram o "agir" de Enfermagem mais próximo da realidade vigente. Admite-se que o presente estudo possui limitações por dissertar apenas a visão de discentes sobre a metodologia empregada, assim, incentiva-se o emprego frequente desse tipo de metodologia nas instituições e mais estudos sobre o tema Leptospirose.

Palavras-chave: Leptospirose, Diagnóstico de Enfermagem, Educação em Enfermagem.

\begin{abstract}
Objective: To report the experience of undergraduates in Nursing School in the construction of Nursing Diagnostics for a case study of patients with Lepstospirosis. Methods: This is a descriptive, qualitative, experience-type study carried out during the course "Infectious and Parasitic Diseases" of the Undergraduate Nursing course of a Public University in Belém-PA. The students selected a theme about the pathologies prevalent in the Amazon, for perform the Nursing Diagnostics. The authors were commissioned to carry out the diagnoses for the Leptospirosis pathology through clinical examination of the patient. Results: From the case study proposed in the classroom, the following "Nursing Diagnostics" based on NANDA-I were selected: Risk for infection; Risk of ineffective thermoregulation; Impaired walking; Unbalanced nutrition: less than bodily needs; Acute pain. Final considerations: The use of active methodologies in educational institutions contributes to the critical-reflective formation of future Nurses, who will be better able to develop the Nursing "action" closer to the current reality. It is admitted that the present study has limitations because it only teaches the students' vision about the methodology used, thus, it is encouraged the frequent use of this type of methodology in the institutions and more studies on the theme Leptospirosis.
\end{abstract}

Key words: Leptospirosis, Nursing Diagnosis, Education, Nursing.

\footnotetext{
1Universidade do Estado do Pará (UEPA), Belém-Pará. *E-mail: glenda.quemel@gmail.com
} 


\section{RESUMEN}

Objetivo: Informar la experiencia de graduados en Enfermería en la construcción de Diagnósticos de Enfermería para un estudio de caso de paciente con Leptospirosis. Métodos: Se trata de un estudio descriptivo, cualitativo, tipo relato de experiencia, realizado durante la disciplina "Enfermedades Infecciosas y Parasitarias" del curso de Graduación en Enfermería de una Universidad Pública, en Belém-PA. Los discentes seleccionaron un tema sobre las patologías prevalentes en la Amazonia, para realizar los Diagnósticos de Enfermería. Las autoras quedaron encargadas de realizar los diagnósticos para la patología Leptospirosis mediante examen clínico del paciente, transcrito en el papel de sorteo. Resultados: Del estudio de caso propuesto en el aula, se seleccionaron los siguientes "Diagnósticos de enfermería" basados en NANDA-I: Riesgo de infección; Riesgo de termorregulación ineficaz; Caminar deteriorado; Nutrición desequilibrada: menos que las necesidades corporales; Dolor agudo. Consideraciones finales: La utilización de metodologías activas en las instituciones de enseñanza contribuye a la formación crítica-reflexiva de futuros enfermeros, los cuales estarán más aptos para desarrollar el "actuar" de Enfermería más cercano a la realidad vigente. Se admite que el presente estudio tiene limitaciones por disertar sólo la visión de los discentes sobre la metodología empleada, así, se incentiva el empleo frecuente de ese tipo de metodología en las instituciones y más estudios sobre el tema Leptospirosis.

Palabras clave: Leptospirosis, Diagnóstico de Enfermería, Educación en Enfermería.

\section{INTRODUÇÃO}

O clima tropical brasileiro, a vulnerabilidade socioeconômica e as condições sanitárias inadequadas favorecem o surgimento de diversas doenças, dentre elas está a leptospirose. A leptospirose é uma zoonose endêmica e epidêmica que surge principalmente em períodos chuvosos, transmitida pela bactéria denominada leptospira, sendo um importante problema de saúde pública devido à alta incidência em países em desenvolvimento (BRASIL, 2014).

No Brasil, nos anos entre 2000 a 2010, foram relatados que $80 \%$ das pessoas que moram em locais de enchentes ou que laboram em atividades de limpezas de caixa d' agua, fossas e valas, além de catadores de lixo reciclável e garis, foram acometidos por essa patologia, enquanto que $10 \%$ foram a óbito e $50 \%$ desenvolveram hemorragia pulmonar. As leptospiras podem habitar água estagnada, solo úmido, matéria orgânica em decomposição, plantas, animais e o homem. Os principais animais disseminadores desta doença no meio urbano são os roedores, os quais são reservatórios crônicos e os responsáveis pela dispersão das bactérias leptospiras no ambiente (BARACHO JM et al., 2017).

As leptospiras penetram o organismo humano por meio de lesões cutâneas, membranas mucosas oral, nasal ou conjuntivas. Após a penetração, as bactérias alcançam a corrente sanguínea e podem atingir a todos os órgãos, acometendo principalmente o fígado, rins, coração e músculo esquelético; e seu período de incubação varia entre 1 a 30 dias. Dentre os principais sintomas estão: cefaleia, febre de instalação abrupta, mal-estar e mialgia, principalmente nos músculos que formam o tríceps sural tais como: gastroquinêmios lateral e medial, e sóleo com inserções no calcâneo pelo tendão de Aquiles (BARACHO JM et al., 2017; BRASIL, 2014).

Nesse contexto, a construção da Enfermagem como Ciência, ao longo da história, vem evoluindo com intuito de gerar profissionais autônomos, a partir de uma assistência organizacional com o cuidado sistematizado, a fim de respeitar as singularidades de cada indivíduo, objetivando um atendimento de qualidade, sensível e resolutivo para promover, prevenir e recuperar (OLIVEIRA CS, BORGES MS, 2017).

O Processo de Enfermagem (PE) é uma ferramenta crítica privativa do profissional enfermeiro e utilizada para conferir uma assistência de enfermagem adequada ao paciente, em que se aplicam as melhores evidências no cuidar dos indivíduos em resposta a sua saúde e à doença (POTTER P, PERRY A, 2013).

O PE é dinâmico, contínuo e possui contém cinco etapas: histórico, diagnóstico, prescrição, implementação e avaliação. No presente manuscrito, destaca-se a segunda etapa caracterizada pelo diagnóstico de enfermagem definido como um julgamento clínico baseado em informações, ou seja, é um 
julgamento clínico sobre as respostas do indivíduo, da família ou da comunidade para os problemas de saúde reais ou potenciais, para a partir disso selecionar intervenções mais eficazes (POTTER P, PERRY A, 2013).

Atualmente, existem quatro tipos de Diagnósticos de Enfermagem usados mundialmente, que são o Diagnóstico com foco no problema, que segundo NANDA-Internacional (2018), é "um julgamento clínico a respeito de uma resposta humana indesejável a uma condição de saúde/processo da vida que existe em um indivíduo, família, grupo ou comunidade", ou seja, é uma condição clínica existente e observada pelo Enfermeiro; o Diagnóstico de Risco, o qual em consonância NANDA-Internacional (2018), é "um julgamento clínico a respeito da suscetibilidade de um indivíduo, família, grupo ou comunidade para o desenvolvimento de uma resposta humana indesejável a uma condição de saúde/processo da vida", ou seja, é uma condição que poderá acontecer; o Diagnóstico de Promoção à Saúde, que de acordo com NANDA-Internacional (2018), é "um julgamento clínico a respeito da motivação e do desejo de aumentar o bem-estar e alcançar o potencial humano de saúde", ou seja, é pautado nas repostas de desejo para a mudança de um comportamento; e, por fim, o Diagnóstico de Síndrome, é caracterizado, conforme NANDA-Internacional (2018), como "julgamento clínico relativo a um determinado agrupamento de Diagnósticos de Enfermagem que ocorrem juntos, sendo mais bem tratado por meio de intervenções similares", porém estes dois últimos não foram utilizados neste estudo.

De maneira geral, os Diagnósticos de Enfermagem possuem um título, uma definição, uma característica definidora e um fator relacionado, os quais, dependendo do tipo de diagnóstico e de sua construção, podem ou não ter esses itens citados. Neste sentido, o título é uma frase ou termo que representa o tema do diagnóstico; a definição clareia e delineia o sentido do diagnóstico; as características definidoras são as evidências clinicas (sinais e sintomas) testemunhadas pelo Enfermeiro somente nos Diagnósticos de foco no problema, bem-estar e síndrome; o fator relacionado são as situações clínicas que antecedem, contribuem ou ameaçam o estado de vida do paciente, e está presente somente no Diagnóstico de risco (NANDA-I, 2018).

Para operacionalizar O PE, facilitar e organizar o trabalho da enfermagem, deve-se aplicar a Sistematização da Assistência de Enfermagem (SAE), que segundo Krauzer IM et al. (2015), constitui um instrumento de cuidado, que tem sido colocado como uma possibilidade de oferecer uma identidade à Enfermagem, permitindo ao Enfermeiro aplicar a SAE nos diversos cenários da assistência, tendo como objetivos principais ser desenvolvida de forma a qualificar a assistência e minimizar os riscos aos usuários. É um processo de pensamento crítico que possibilita aplicar melhores evidências disponíveis ao cuidado (ANDRADE YNL et al., 2016).

Em consonância a Krauzer IM et al. (2015), existem algumas propriedades que devem existir para avaliação dessas cinco fases do PE e suas respectivas complexidades, tais como: propriedade propositada, metódica ou sistemática, ativa ou dinâmica, interativa, flexível, e deve basear-se também em Teorias de Enfermagem. A importância da implementação do PE através da SAE é reforçada pelo COFEN por meio de resoluções, pelas quais se determina que o PE deva ocorrer obrigatoriamente em todas as Instituições de Saúde, sendo registrada no prontuário do paciente com detalhamento de cada fase de sua execução.

No cenário brasileiro, o estudo sobre a SAE, destacou-se somente no final de 1980, através do Decreto no 94.406/87, regulamentado pela Lei 7.498/86 do Exercício do Profissional de Enfermagem no País, reforçado pela Resolução 358/2009, do Conselho Federal de Enfermagem (COFEN), que dispõe da implementação da $\mathrm{SAE}$, considerando que o profissional em Enfermagem deve organizar o trabalho diante do método, de sua equipe e dos instrumentos, operacionalizando o PE, a fim de alcançar os objetivos supracitados (KRAUZER IM et al., 2015).

Devido à grande relevância de se conhecer a aplicação da SAE e do PE, assim como as habilidades necessárias para sua realização, faz-se necessário a compreensão dentro da academia sobre essa prática, uma vez que, o acadêmico de Enfermagem na posição de um futuro profissional, deve exercitar esses conhecimentos e habilidades, a fim de ter pensamento crítico e hábil para as situações da realidade de cada pessoa e dessa forma construir e entender a importância de sua autonomia dentro da equipe de saúde (KRAUZER IM et al., 2015). 
No entanto, observa-se que a grande maioria dos acadêmicos de Enfermagem não desenvolve a prática da operalização do PE com a SAE durante as realizações de suas tarefas obrigatórias exigidas pelas Universidades. Segundo Andrade YNL et al. (2016), essa realidade se dá por alguns motivos específicos, tais como as divergências metodológicas dos docentes, a padronização do ensino repassado e o desconhecimento de docentes, o que reflete em um déficit da aplicabilidade dessa ferramenta por parte dos acadêmicos desde 0 início da graduação.

Por sua vez, as metodologias ativas constituem-se como um modelo de formação profissional mais adequado para a realidade, uma vez que, por meio de situações problemas, encontra-se um estímulo para a busca de novos conhecimentos e habilidades, já que, é um processo que tem como característica principal colocar o aluno como responsável por sua própria aprendizagem. Em um estudo realizado por Mesquita SKDC et al., (2016), os desafios para introduzir essas ferramentas ativas no ensino da Enfermagem em uma Universidade Pública perpassam pelos problemas curriculares, os quais funcionam como empecilho para aplicação da metodologia de ensino/aprendizagem, ou seja, grandes divergências entre os componentes curriculares no ensino da Enfermagem trazem ao docente dificuldades para implementar as metodologias ativas em seu cotidiano, surgindo assim, outro desafio que consiste na resistência de docentes em implementar os métodos ativos de ensino/aprendizagem.

A aplicabilidade desse método de aprendizagem no ensino da Enfermagem também está relacionada ao entendimento dessa nova tendência pedagógica por parte dos docentes e o adequado conhecimento desses métodos pelos docentes está diretamente relacionado ao sucesso de sua utilização. Sendo assim, é necessário que as universidades estimulem seus docentes à busca de novos caminhos pedagógicos para ensinar e aprender, que os levem às reflexões críticas a respeito do ensino/aprendizagem que está sendo ministrado no ensino da Enfermagem (MESQUITA SKDC et al., 2016).

Dentre as principais metodologias ativas, pode-se citar a Aprendizagem Baseada em Problemas (PBL), os Grupos Operários, o processo incidente, o estudo de caso, os mapas conceituais, os mapas mentais, as simulações, a metodologia da problematização, o estudo de caso, o estudo dirigido e entre outras, que podem ser utilizadas em sala de aula. Nessa perspectiva, o estudo de caso é entendido como uma abordagem de aprendizagem ativa baseada em situações de contexto real, que pretende desenvolver habilidades e competências ao demonstrar um caso real ou hipotético com várias soluções ou pontos que devem ser percebidos pelos discentes para estimular a investigação científica e a localizar informações que preparem o futuro profissional para atuar na condição demonstrada no caso (MOREIRA JR, RIBEIRO JBP, 2016).

Nesse sentido, abre-se um questionamento que norteará a relevância e discussão do tema proposto: "Qual a experiência dos acadêmicos de Enfermagem na construção de Diagnósticos de Enfermagem para um estudo de caso hipotético de um paciente com Leptospirose?". Por esse motivo justifica-se a investigação dessa problemática, tendo como objetivo principal relatar a experiência de acadêmicas de Enfermagem na construção de Diagnóstico de Enfermagem para um estudo de caso hipotético de paciente com Lepstospirose.

\section{MÉTODOS}

Trata-se de um estudo descritivo, com abordagem qualitativa, do tipo relato de experiência, realizado durante a disciplina "Doenças Infecciosas e Parasitárias" do curso de Graduação em Enfermagem de uma Universidade Pública, no município de Belém-PA.

Os discentes do oitavo período do ano de 2018 desse curso, foram orientados por um docente a selecionarem, por meio de um sorteio, alguns temas do componente curricular. Os temas se referiam às patologias prevalentes na Amazônia, as quais foram descritas em forma de estudo de caso hipotético com o exame clínico transcrito no papel de sorteio, para estas doenças os discentes deveriam realizar os Diagnósticos de Enfermagem.

Esta atividade proposta pelo docente em sala de aula diz respeito à introdução das metodologias ativas no componente curricular da Universidade Pública em questão desde 2011, para facilitar o ensinoaprendizagem dos discentes por meio de situações-problemas, como o estudo em questão. Nesta 
perspectiva, as metodologias podem ser aplicadas pelos professores aos alunos de forma individualizada ou socializada. O método individualizado é proposto por meio da execução de tarefas, a exemplo o estudo dirigido.

O método de ensino socializado é utilizado por meio de trabalhos em grupos, como o estudo de caso, o qual tem por objetivo estimular a integração entre os educandos e promover o desenvolvimento da capacidade de interação interpessoal para que os alunos aprendam a expressar e defender suas ideias (PAIM AS et al., 2015; MESQUITA SKDC et al., 2016).

\section{RESULTADOS}

Diante do estudo de caso proposto em sala de aula, as autoras ficaram incumbidas de realizar os diagnósticos para a patologia Leptospirose mediante o exame clínico do paciente (Quadro 1).

Quadro 1 - Exame clínico obtido pelo sorteio em sala de aula.

Identificação: Paciente do gênero masculino, 29 anos, proveniente do interior do Pará, casado, sem filhos, laborava como gari, evangélico.

Dados socioeconômicos: possui ensino fundamental completo, residência com quatro cômodos, saneamento básico adequado e coleta seletiva duas vezes na semana.

Queixa principal: Internou na Unidade de Terapia Intensiva de um hospital público do município de BelémPA relatando dores no corpo, principalmente, no músculo tríceps sural (panturrilha) bilateralmente, cefaleia intensa, sensação de mal estar geral, astenia e febre; paciente relatou que seu estado de saúde pode ser devido à falta de uso de Equipamento de Proteção Individual (EPI), como botas e luvas durante seu trabalho.

Diagnóstico médico: Leptospirose.

Antecedentes mórbidos pessoais: Tabagista e etilista por cinco anos. Nega alergias e qualquer patologia. Antecedentes mórbidos familiares: Nega qualquer comorbidade familiar e relata que familiares faleceram por causas naturais.

Ao exame físico: Paciente consciente e orientado, normotenso (120x90 mmhg), normocárdico (72 bpm), eupneico (18 rpm) e febril $\left(38^{\circ} \mathrm{C}\right)$. Tórax em tonel, sem alterações visíveis, porém paciente queixou-se de dores e relatou dificuldade para dormir, $\mathrm{BCNF} / 2 \mathrm{~T}$, murmúrios vesiculares presentes sem ruídos adventícios. Abdômen globoso, sem alterações visíveis, $\mathrm{RHA}+$, dieta livre, paciente relatou falta de apetite e preferia não comer. Quanto aos membros inferiores (MMII) e membros superiores (MMSS), sem alterações visíveis e acesso venoso periférico em MSD recebendo tratamento medicamentoso de 8/8 horas e solução fisiológica a $0,9 \%$, e deambulante com dificuldade por conta de dores. Funções fisiológicas de eliminação presentes e espontâneas.

Fonte: Autoria Própria, 2019.

A partir do caso hipotético proposto em sala de aula, foram realizados os "Diagnósticos de Enfermagem" e os discentes utilizaram a classificação da NANDA-Internacional (NANDA-I, 2018) para a construção destes diagnósticos (Quadro 2). 
Quadro 2 - Diagnósticos de Enfermagem para o estudo de caso de paciente com Leptospirose.

\begin{tabular}{|c|c|c|c|c|}
\hline Título & $\begin{array}{l}\text { Caracteristica } \\
\text { definidora }\end{array}$ & Fatores de riscos & Domínio & $\begin{array}{c}\text { Tipo de } \\
\text { diagnóstico }\end{array}$ \\
\hline $\begin{array}{l}\text { 1.Risco para } \\
\text { infeç̧ão. }\end{array}$ & 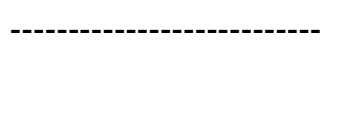 & $\begin{array}{c}\text { Relacionado ao } \\
\text { acesso venoso } \\
\text { periférico em MSD. }\end{array}$ & $\begin{array}{l}\text { Domínio 11: } \\
\text { Segurança/proteção }\end{array}$ & Risco \\
\hline $\begin{array}{l}\text { 2.Risco de } \\
\text { termoregulação } \\
\text { ineficaz. }\end{array}$ & 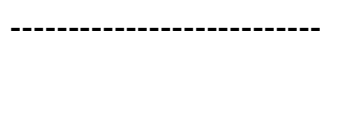 & $\begin{array}{l}\text { Relacionado à } \\
\text { temperatura acima da } \\
\text { faixa normal. }\end{array}$ & $\begin{array}{l}\text { Domínio 11: } \\
\text { Segurança/proteção }\end{array}$ & Risco \\
\hline $\begin{array}{l}\text { 3.Deambulação } \\
\text { prejudicada. }\end{array}$ & $\begin{array}{l}\text { Caracterizado por } \\
\text { limitação da } \\
\text { movimentação } \\
\text { independente, a pé, } \\
\text { pelo ambiente. }\end{array}$ & $\begin{array}{l}\text { Relacionado ao } \\
\text { comprometimento do } \\
\text { músculo sural. }\end{array}$ & $\begin{array}{c}\text { Domínio 4: } \\
\text { Atividade/repouso }\end{array}$ & $\begin{array}{l}\text { Diagnóstico } \\
\text { com foco no } \\
\text { problema }\end{array}$ \\
\hline $\begin{array}{l}\text { 4.Nutrição } \\
\text { desequilibrada: } \\
\text { menor do que } \\
\text { as } \\
\text { necessidades } \\
\text { corporais. }\end{array}$ & $\begin{array}{l}\text { Caracterizada pela } \\
\text { ingestão insuficiente } \\
\text { de nutrientes para } \\
\text { satisfazer as } \\
\text { necessidades } \\
\text { metabólicas. }\end{array}$ & $\begin{array}{l}\text { Relacionado à } \\
\text { dificuldade na } \\
\text { ingestão de calorias } \\
\text { suficientes. }\end{array}$ & Domínio 2: Nutrição & $\begin{array}{l}\text { Diagnóstico } \\
\text { com foco no } \\
\text { problema }\end{array}$ \\
\hline 5.Dor aguda. & $\begin{array}{l}\text { Caracterizada pelo } \\
\text { relato de dor, } \\
\text { alteração no apetite } \\
\text { e nos padrões de } \\
\text { sono e repouso, } \\
\text { com duração menor } \\
\text { que } 3 \text { meses. }\end{array}$ & $\begin{array}{l}\text { Relacionado à agente } \\
\text { biológico lesivo. }\end{array}$ & $\begin{array}{l}\text { Domínio 12: } \\
\text { Conforto }\end{array}$ & $\begin{array}{l}\text { Diagnóstico } \\
\text { com foco no } \\
\text { problema }\end{array}$ \\
\hline
\end{tabular}

Fonte: Autoria Própria, 2019.

\section{DISCUSSÃo}

Nessa experiência, os discentes realizaram dois Diagnósticos de risco e três Diagnósticos com foco no problema. Quanto aos Diagnósticos de risco, o primeiro foi o de risco de infecção (Diagnóstico 1), o qual pertence ao Domínio Segurança e Proteção da NANDA-I (classe 1, 00004), este foi selecionado pelo fato de que o exame clínico hipotético transcrito relatava que o paciente recebia medicação por acesso venoso periférico em membro superior direito (MSD). Esse procedimento é considerado invasivo por causar descontinuidade da pele pela introdução de acesso venoso e constitui-se um fator de risco para infecções de corrente sanguínea por conta da permanência do acesso venoso periférico por mais de 72 horas, pois para Johann DA et al. (2016) o risco de infecção na corrente sanguínea aumenta após esse tempo, o que Danski MTR et al. (2016) corrobora e soma ao ilustrar que existem outros riscos em virtude dessa permanência, tais como a flebite, a infiltração e a obstrução da veia periférica, sobretudo, se a veia utilizada for menos calibrosa. Nesta fenda, o Diagnóstico de Enfermagem Risco de Infecção é conceituado por Silva RAR et al. (2016) como o "estado iminente de albergar alguma injúria biológica, como vírus, bactéria e fungos, ocasionada por procedimentos invasivos, ou não, que afetam assim, o sistema imunológico do cliente".

O Diagnóstico de risco de termoregulação ineficaz (Diagnóstico 2), também pertencente ao Domínio Segurança e Proteção da NANDA-I (classe 6, 00274), é entendido como predisposição à mudança da temperatura corporal, assim, os discentes o escolheram por atrelarem ao fato de que, no contexto, o paciente possuía estado de pirexia $\left(38^{\circ} \mathrm{C}\right)$ que se acredita estar relacionado à presença do microorganismo causador da Lepstopirose no organismo humano. É importante destacar que a temperatura corporal está em constante 
equilíbrio entre o meio interno e o externo, a pirexia acontece quando os neurônios sensíveis à temperatura presentes na pele, na medula espinal, nas vísceras abdominais e entre outros locais, enviam sinais de desequilíbrio para o centro regulatório da temperatura, localizado no hipotálamo, cuja resposta manifesta-se por esse quadro; logo é necessário monitoramento constante (BEIRÃO EF et al., 2017).

No que tange aos Diagnósticos com foco no problema, escolheu-se o de deambulação prejudicada (Diagnóstico 3), pertencente ao Domínio Atividade e Repouso do NANDA-I (classe 2, 00088), por corresponder à uma limitação da capacidade de andar e, no caso do hipotético paciente, essa limitação foi associada pelos discentes à queixa principal de dor intensa e aguda nos músculos tríceps sural, muito característico da patologia Leptospirose. Nesse sentido, em um estudo realizado em um município de área irregular e sem saneamento básico de Porto Alegre com uma amostra de 89 moradores, demonstrou que a população em geral possui lacunas e dúvidas sobre saúde ambiental em que apenas $60 \%$ realiza separação do lixo e $40 \%$ já haviam tido Leptospirose, o que constitui-se uma problemática frente a esses dados alarmantes, além de que o paciente deste estudo em questão laborava como gari, ficando claro que as condições urbanas podem afetar diretamente o seu estado de vida. Galdino SJ, Malysz ST (2016), corroboram que os garis estão expostos constantemente a acidentes de trabalho e a contaminação por agentes causadores de doença e, para tanto, é imprescindível a educação ambiental, os investimentos públicos em saneamento e obrigatoriedade de Equipamentos de Proteção Individual, como óculos, luvas de borracha, botas, máscaras e protetores auricular.

Em relação ao Diagnóstico de Nutrição desequilibrada: menos do que as necessidades corporais (Diagnóstico 4), pertencente ao Domínio Nutrição (classe 1, 00002), este é compreendido como a ingestão de nutrientes insuficiente. No momento deste estudo, foi levantada a hipótese, por parte do docente, de que a falta de apetite e desejo de não se alimentar fosse motivado pelo quadro de dor aguda ou pelos sentimentos negativos em relação à internação. Este fato corrobora com Tavares AT e Pawlowytch PWM (2013), pois para estes autores qualquer quadro patológico seguido de internação pode abalar o estado emocional e psicológico do cliente devido a sentimentos como a vulnerabilidade, a despersonalização e a ansiedade, que se manifestam pelo humor irritável, estresse, depressão e falta de apetite, e devem ser trabalhados de forma multidisciplinar por Enfermeiros, Psicólogos, Médicos e Terapeutas Ocupacionais, na lógica interdisciplinar.

Por fim, o Diagnóstico de Dor Aguda (Diagnóstico 5), pertencente ao Domínio de Conforto (classe 1, 00132), entendido como experiência sacal, única e multifacetada em decorrência a lesão real ou potencial de início súbito ou lento, de intensidade leve à intensa com duração menor de 3 meses, foi realizado pelos discentes devido a situação problema indicar que o paciente relatava dor em vários grupos musculares e por ser considerada como um quinto (5ํ) sinal vital. Para Freitas RL e Silva JA (2018) em consonância com Araújo LC e Romero B (2015), o profissional de saúde deve avaliar a intensidade, a característica, os fatores de melhora e de piora, e o tempo de surgimento da sensação dolorosa em todos os pacientes; e ressalta-se que, apesar da dor ser um efeito subjetivo, é passível de avaliações objetivas por meio de instrumentos como o Questionário desenvolvido por Melzack em 1975 na Universidade de McGill, a escala visual numérica, a escala analógica visual, a escala quantitativa não numérica, a escala de mãos e a escala comportamental.

\section{CONSIDERAÇÕES FINAIS}

Diante da metodologia ativa apresentada aos discentes para que aplicassem seus conhecimentos, notouse a satisfação da turma ao realizar esse tipo de atividade educativa, tendo em vista que a metodologia coloca o aluno no centro do processo de aprendizado, valoriza seus conhecimentos e horizontaliza a educação. Acredita-se que a utilização desse tipo de ensino nas instituições de ensino de Graduação em Enfermagem contribui positivamente para a formação crítica-reflexiva dos futuros Enfermeiros, os quais estarão mais aptos a desenvolveram o "agir" de Enfermagem muito mais próximo da realidade vigente. Admite-se que o presente estudo possui limitações por dissertar apenas a visão de discentes sobre a metodologia empregada e suas capacidades de articular os conhecimentos apreendidos com a realidade proposta, assim, incentiva-se 0 emprego frequente desse tipo de ensino nas instituições de nível superior e mais estudos sobre o tema Leptospirose, que é uma doença negligenciada, porém frequente por conta da infraestrutura urbana brasileira.

REAS/EJCH | Vol. Sup.25 | e826 | DOI: https://doi.org/10.25248/reas.e826.2019 Página 7 de 8 


\section{REFERÊNCIAS}

1. BRASIL. Ministério da Saúde. Secretaria de Vigilância em Saúde. Departamento de Vigilância das Doenças Transmissíveis. Leptospirose: diagnóstico e manejo clínico / Ministério da Saúde, Secretaria de Vigilância em Saúde. Departamento de Vigilância das Doenças Transmissíveis. - Brasília: Ministério da Saúde, 2014.

2. BARACHO JM et al. Incidência de casos de leptospirose humana em Pernambuco: uma análise dos dados epidemiológicos de 2015. Caderno de Graduação-Ciências Biológicas e da Saúde-FACIPE, v. 3, n. 2, p. $19,2017$.

3. OLIVEIRA CS, BORGES MS. Representações sociais de Enfermeiros que cuidam de crianças sobre a sistematização da assistência de Enfermagem. Revista Gaúcha de Enfermagem. 2017; 38(3): e66840.

4. POTTER P, PERRY A. Fundamentos de Enfermagem. 8.ed. Rio de Janeiro: Elsevier, 2013.

5. NANDA-Internacional. HERDMAN TH, KAMITSURU S (Ed.). NANDA International, Inc. Nursing Diagnoses: Definitions \& Classification 2018-2020. Thieme, 2018.

6. KRAUZER IM et al. Sistematização da assistência de Enfermagem na atenção básica: o que dizem os Enfermeiros?.Ciencia y Enfermeria. 2015; 21(2): 31-38.

7. ANDRADE YNL et al. Conhecimento de acadêmicos de Enfermagem sobre o ensino-aprendizagem da sistematização da assistência de Enfermagem. Rev Rene. 2016; 17(15): 602-609.

8. MESQUITA SKDC et al. Metodologias ativas de ensino/aprendizagem: dificuldades de docentes de um curso de enfermagem. Trabalho, Educação e Saúde, v. 14, n. 2, p. 473-486, 2016.

9. PAIM AS et al. Metodologias de ensino utilizadas por docentes do curso de enfermagem: enfoque na metodologia problematizadora. Enfermería Global, v. 14, n. 1, p. 136-169, 2015.

10. MOREIRA JR, RIBEIRO JBP. Prática pedagógica baseada em metodologia ativa: aprendizagem sob a perspectiva do letramento informacional para o ensino na educação profissional. Periódico Científico Outras Palavras, volume 12, número 2, ano 2016, página 100.

11. JOHANN DA et al. Fatores de risco para complicações no cateter venoso periférico em adultos: análise secundária de ensaio clínico randomizado. Revista Latino Americana de Enfermagem. 2016;24e: 2833.

12. DANSKI MTR et al. Complicações relacionadas ao uso do cateter venoso periférico: ensaio clínico randomizado. Acta Paul Enferm. 2016; 29(1): 84-92.

13. SILVA RAR et al. Diagnósticos, resultados e intervenções de Enfermagem para pacientes em diálise peritoneal. Acta Paul Enferm.2016;29 (5):486-93.

14. BEIRÃO EF et al. Fundamentos da termorregulação para hidroterapia. Rev. Ibirapuera. 2017; 13(1): $62-70$.

15. GALDINO SJ, MALYSZ ST. Os riscos ocupacionais dos garis coletores de resíduos sólidos urbanos os riscos ocupacionais dos garis coletores de resíduos sólidos urbanos. Revista Percurso. 2016;8(2):187-205.

16. TAVARES AT, PAWLOWYTCH PWM. Percepção dos pacientes sobre sua permanência em uma unidade de terapia intensiva. Rev Saúde Meio Ambient. 2013; 2(2):32-43.

17. FREITAS RL, SILVA JA. Os desafios de mensurar a dor. Br J Pain. 2018; 1(1):2-3.

18. ARAÚJO LC, ROMERO B. Dor: avaliação do 5osinal vital. Uma reflexão teórica. Rev Dor. 2015; 16(4):291-296. 